\title{
Tratamento endovascular em paciente portador de coarctação da aorta: relato de caso
}

\author{
Endovascular treatment in a patient with aortic \\ coarctation: case report
}

\begin{abstract}
Eugênio Carlos Almeida Tinoco, Antônio Carlos Botelho, Bruno Baião Luquini, Rafael Lima Campanha, Marcelo Nascimento, Luciana Abreu Horta, Daniela Mendonça Sueth, Felipe Montes Pena, Lincoln Rangel de Medeiros Teixeira*
\end{abstract}

\section{Resumo}

Coarctação da aorta constitui um estreitamento de origem congênita na porção inicial da aorta torácica. Tem incidência de 6 a $8 \%$ em nascidos vivos. A apresentação clínica é variada. O tratamento de escolha é o cirúrgico. Relatamos um caso de paciente do sexo feminino de 31 anos, com queixa de dor constrictiva na região cervical, dispnéia e claudicação intermitente em membros inferiores, sendo diagnosticada coarctação da aorta associada a estenose aórtica grave, que foi tratada com combinação das técnicas implantação de stent endovascular e angioplastia por balão.

Palavra-chave: Coarctação aorta, stent, angioplastia.

\section{Introdução}

A coarctação da aorta (CoA) é responsável por 6 a $8 \%$ dos nascidos vivos com cardiopatia congênita. Constitui uma das doenças mais freqüentes entre as anomalias obstrutivas do ventrículo esquerdo. Quando não tratada, a CoA tem mau prognóstico, com a maioria dos pacientes indo a óbito antes dos 40 anos. $\mathrm{O}$ tratamento cirúrgico, tradicionalmente, é a opção terapêutica escolhida, com bons resultados na evolução ${ }^{1-3}$.

Os stents foram introduzidos na década de 90 , como terapia adjunta à angioplastia com balão, com objetivo de minimizar as complicações oriundas desse último procedimento isolado. É uma técnica segura e eficaz, associada a bons resultados a curto e médio prazo, reduzindo os índices de complicações ${ }^{1-3}$. Este artigo

\footnotetext{
* Serviço de Cirurgia Vascular e Endovascular, Hospital São José do Avaí, Itaperuna, RJ.
}

Artigo submetido em 14.10.06, aceito em 08.01.07.

J Vasc Bras 2007;6(1):82-85.

Copyright $\odot 2007$ by Sociedade Brasileira de Angiologia e de Cirurgia Vascular

\begin{abstract}
Aortic coarctation is a congenital stenosis in the initial portion of the thoracic aorta. Its incidence ranges between $6-8 \%$ of liveborns. Clinical presentations are diversified. The treatment of choice is surgery. We report the case of a 31-year-old female patient with constrictive pain in the cervical region, dyspnea, and intermittent claudication of the lower limbs. She was diagnosed with aortic coarctation associated with severe aortic stenosis, which was treated using a combination of endovascular stent implantation and balloon angioplasty.
\end{abstract}

Keywords: Aortic coarctation, stent, angioplasty.

mostra tratamento combinado pelas técnicas citadas acima, em paciente adulta com CoA.

\section{Relato do caso}

Paciente do sexo feminino, 31 anos, casada, foi encaminhada ao serviço de cirurgia vascular e endovascular do Hospital São José do Avaí com diagnóstico de CoA. Relatou que há 8 meses iniciou quadro de dor constrictiva na região cervical de intensidade intermediária, sem irradiação ou fatores atenuantes ou agravantes, associada à dispnéia após moderados esforços, com evolução atual para pequenos esforços, e claudicação intermitente para curtas distâncias. Apresentava hipertensão arterial, mas fazia uso irregular do medicamento.

$\mathrm{Na}$ ausculta cardíaca, apresentava sopro sistólico mais audível em foco aórtico (3+/6+), com irradiação para as carótidas. A pressão arterial encontrava-se discrepante entre os membros. O exame vascular demonstrava membros superiores com pulsos simétricos e 
de amplitude adequada e membros inferiores com pulsos impalpáveis e membros normotérmicos e acianóticos. O restante dos sistemas apresentava-se normal.

À internação, portava resultados dos seguintes exames:

1. Eco-Doppler-cardiograma: estenose aórtica grave, ectasia de aorta descendente e não-localização do segmento proximal da aorta descendente em sua posição habitual;

2. Cinecoronariografia e ventriculografia: estenose aórtica e CoA;

3. Angiotomografia computadorizada helicoidal: CoA no segmento torácico proximal, $1,5 \mathrm{~cm}$ após a emergência da artéria subclávia esquerda, com dilatação pré-estenótica (Figura 1).

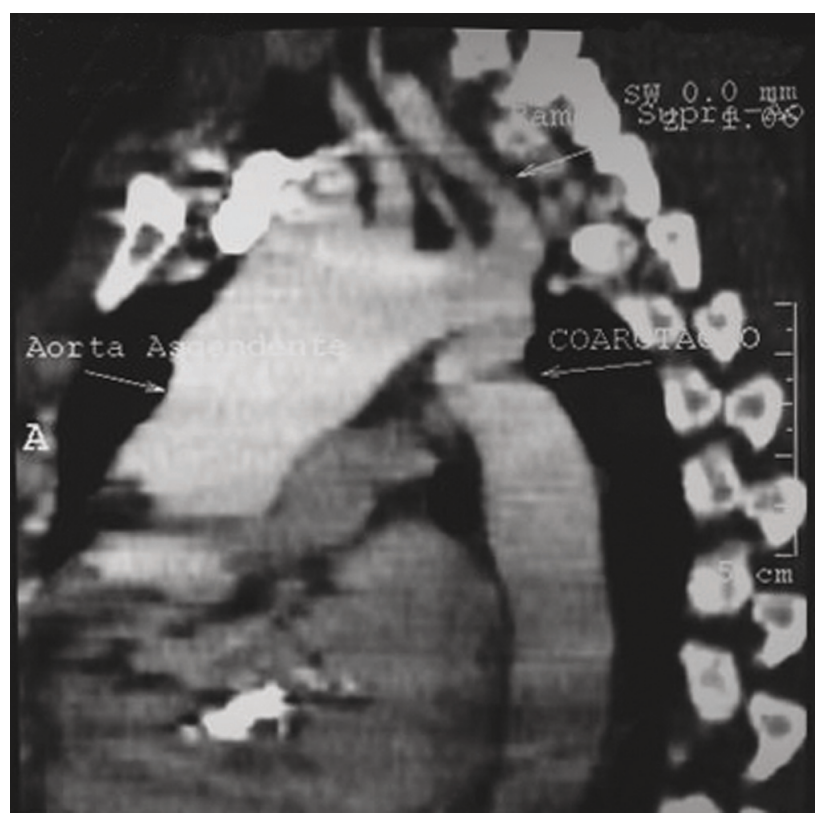

Figura 1 - Angiotomografia computadorizada demonstrando coarctação da aorta em nível de aorta torácica, porção descendente alta

Devido à dilatação pré-estenótica comprometer o óstio das artérias subclávia e carótida comum esquerdas, optamos pela realização de ponte da artéria subclávia direita para as artérias subclávia e carótida esquerdas. A cirurgia foi realizada através de incisão supraclavicular bilateral, com dissecção dos vasos correspondentes. Como conduto, utilizamos prótese de politetrafluoretileno (PTFE) de $8 \mathrm{~mm}$, com localização pré-traqueal e subcutânea da artéria subclávia direita em direção à artéria subclávia esquerda. Para a artéria carótida comum esquerda, foi confeccionada uma ramificação a partir da prótese de PTFE com outro segmento de prótese do mesmo material e medida. Sendo assim, os dois vasos - artérias carótida comum e subclávia esquerdas - foram ligados em sua origem. Optou-se, então, pelo uso de stent recoberto pela presença de dilatação pré-estenótica significativa, onde, no segundo tempo, foi realizada dissecção da artéria femoral comum direita e implante de endoprótese Zenith $32 / 80 \mathrm{~mm}$, posteriormente dilatada com balão de $32 \mathrm{~mm}$, procedimento este realizado com sucesso. Porém, durante a retirada da bainha da endoprótese, houve ruptura da artéria femoral comum direita, sendo prontamente corrigida com acesso à artéria ilíaca comum direita por via retroperitoneal e colocação de enxerto entre as artérias ilíaca comum direita e femoral comum direita, utilizando prótese de PTFE de $8 \mathrm{~mm}$ de diâmetro (Figura 2).

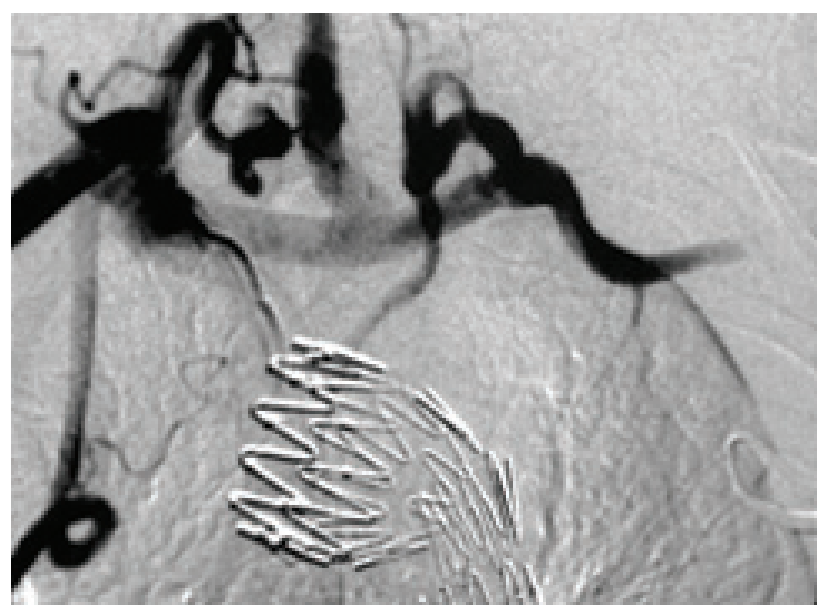

Figura 2 - Aspecto final demonstrando o bypass realizado entre artéria subclávia direita e subclávia e carótida comum esquerdas e a endoprótese colocada no local da coarctação

A paciente recebeu alta no quinto dia, com pulsos simétricos e de alta amplitude, sem discrepância da pressão arterial entre os membros, e foi encaminhada ao serviço de cardiologia para tratamento da estenose aórtica. 


\section{Discussão}

A CoA consiste, tipicamente, em uma lesão com estreitamento localizado e excêntrico da aorta descendente proximal à extremidade aórtica do canal arterial ou ligamento arterioso. A apresentação clínica varia com a faixa etária na ocasião do diagnóstico. $\mathrm{O}$ achado característico baseia-se na discrepância dos pulsos e da pressão arterial sistólica nos membros superiores e inferiores, porém muitos adultos são assintomáticos. O diagnóstico pode ser feito durante exame físico de rotina, com os achados acima referidos, e haver diminuição ou ausência de pulsos femorais ${ }^{4-6}$.

O tratamento cirúrgico é indicado para pacientes com falência cardíaca na primeira infância e para crianças ou adultos com diagnóstico tardio. $\mathrm{O}$ nãotratamento implica, futuramente, em insuficiência cardíaca, endocardite bacteriana, acidente vascular cerebral e doença coronariana ${ }^{1-7}$.

Em 1948, foi realizada a primeira bem-sucedida correção cirúrgica da CoA por Craaford, com anastomose término-terminal. Desde 1961, foram publicadas mais de 11 variantes técnicas ${ }^{8}$. O desenvolvimento das técnicas de intervenção percutânea, passando pela angioplastia por balão, e, mais recentemente, a implantação de stents endovasculares aumentaram as possibilidades de tratamento minimamente invasivas da doença ${ }^{9}$. As indicações para uso de stents cobertos, balão expansíveis ou auto-expansíveis são: obstrução crítica, definida como diâmetro mínimo no nível da $\mathrm{CoA}<3 \mathrm{~mm}$ à angiografia, associada a atresia de lúmen aórtico, canal arterial patente, alterações degenerativas da parede aórtica, idade acima de 30 a 40 anos, formação de aneurisma após implantação de stents convencionais, detectados agudamente ou no segmento, e presença de fraturas circunferenciais em prótese implantada previamente na aorta $^{1-9}$.

Alguns autores preconizam a dilatação do segmento acometido antes da implantação do stent, enquanto outros defendem o oposto. Entretanto, em alguns casos, a dificuldade na dilatação do segmento estenosado aumenta o risco da expansão inadequada do stent, tornando-o insuficiente, sendo necessária sua remoção cirúrgica ${ }^{7}$.
A angioplastia por balão é uma alternativa segura e efetiva no tratamento da CoA e reflete um sucesso em $93,7 \%$ dos casos, com índice de complicações de 1,8\%, relacionado à idade do paciente e local anatômico da lesão ${ }^{10}$. As principais complicações em curto prazo são hipertensão e coarctação recorrente ou residual, enquanto que, em longo prazo, encontramos aneurisma de aorta ${ }^{6}$. Em relação ao uso de stents, os relatos de segmento imediato e em médio prazo são encorajadores. Os resultados iniciais mostraram resolução parcial ou completa do gradiente de pressão na maioria dos pacientes, sendo melhores do que os obtidos com a dilatação com balão isoladamente. A taxa de mortalidade é menor que 1 a $2 \%$, e as principais complicações incluem grandes dissecções vasculares, ruptura aórtica durante liberação do stent, deslizamento ou fratura do mesmo e sangramento com ou sem formação de hematoma no local do acesso ${ }^{1}$.

Concluímos que a correção cirúrgica sempre foi o procedimento de escolha para a $\mathrm{CoA}$, melhorando a história natural da doença e o controle da hipertensão arterial. O desejo de reduzir a morbimortalidade associada ao procedimento cirúrgico motivou a terapia endovascular para a doença. A angioplastia por balão surgiu como alternativa ao tratamento cirúrgico, no qual, apesar de resultados iniciais satisfatórios, a possibilidade de reestenose sempre ocorria a médio e longo prazo. Com a introdução dos stents vasculares, acredita-se haver uma menor possibilidade de reestenose. Stents recobertos vêm sendo utilizados no manejo da CoA, prevenindo a formação de aneurismas ou dissecções.

\section{Referências}

1. Neves J, Fraga V, Silva R, et al. Uso de stents no tratamento da coarctação da aorta. Rev Bras Cardiol Invas. 2005;13:153-66.

2. Kreutzer RC, Rozenbaum JC, Solsona CE, et al. Resultados quierúrgicos em coarctación de la aorta con anastomosis terminoterminal ampliada. Rev Arg Cardiol. 2003;71:93-101.

3. Benson L, McLaughlin PR. Coarctation of the aorta. In: Freedom R, Yoo SJ, Mikailian H, Williams WG, eds. The natural and modifical history of congenital heart disease. New York: Blackwell; 2004. p. 251-75. 
4. Freedom RM, Mawson JB, Yoo SJ, Benson LN. Coarctation of the aorta. In: Freedom RM, Mawson JB, Yoo SJ, Benson LN, eds. Congenital heart disease. Textbook of angiography. Armonk, NY: Futura; 1997. p. 899-921.

5. Koch VH, Furusawa E, Haber J, Foronda G, Okay Y. Utilização da monitorização ambulatorial de pressão arterial no diagnóstico e no tratamento da coarctação da aorta. J Bras Nefrol. 2000;22:209-13.

6. Brickner ME, Hillis LD, Lange RA. Congenital heart disease in adults. Second of two parts. N Engl J Med. 2000;342:334-42.

7. Alcibar J, Pena N, Onate A, Gochi R, Barrenechea JI. Stent implantation in a adult with coarctation of the aorta in the presence of advanced secondary heart failure. Tex Heart Inst J. 1999;26:143-7.

8. Lorier G, Wender O, Kalil RAK, et al. Coartação de aorta em crianças até um ano. Análise de 20 anos de experiência. Arq Bras Cardiol. 2005;85:51-6.
9. Tostes J, Ferreira R, Baptista C, Anjos R. Deiscência da aorta após colocação de stent - resolução percutânea imediata. Rev Port Cardiol. 2006;25:121-4.

10. Munayer Calderon J, Zabal Cerdeira C, Ledesma Velazco M, et al. Balloon angioplasty in aortic coarctation: a multicentric study in México. Arch Cardiol Mex. 2002;72:20-8.

Correspondência:

Daniela Mendonça Sueth

Rua Apolinário Cunha, 115/501, Bairro Cidade Nova CEP 28300-000 - Itaperuna, RJ

Tel.: (22) 3822.0051, (22) 8115.7230

E-mail:dmsueth@yahoo.com.br 